\title{
Transcatheter aortic valve implantation in nonagenarians: selectively feasible or extravagantly futile?
}

\author{
Antonis S. Manolis, Antonis A. Manolis \\ Third Department of Cardiology, Athens University School of Medicine, Athens, Greece \\ Correspondence to: Antonis S. Manolis, MD. Third Department of Cardiology, Athens University School of Medicine, Vas. Sofias 114, Athens 115 27, \\ Greece. Email: asm@otenet.gr.
}

\begin{abstract}
A growing number of nonagenarians is recorded as life expectancy increases. Unfortunately, this extreme-aged group is plagued by increased prevalence of aortic stenosis amidst a higher occurrence of comorbidities that pose dilemmas to cardiologists and cardiac surgeons when having to choose a conservative or interventional treatment modality, and a surgical or transcatheter aortic valve implantation (TAVI) approach. TAVI is an expensive procedure, which also confers a higher mortality and morbidity risk in nonagenarians, compared to younger patients. Considering the physiologic rather the chronologic age alone, and adopting a shared-decision making approach (participatory medicine), it may be more realistic to determine a patient's candidacy for this non-surgical therapeutic modality. Thus, it comes down to the patient selection process by having the heart team review each nonagenarian case individually and getting the patient and the family involved, always aiming to prolong and improve patient's quality of life (QoL), but also taking into consideration patient preferences and values, sharing and respecting goals, realistic expectations, and end-of-life views and ideas. One should keep in mind that there is always the possibility that TAVI may be clinically futile for patients who have a multitude of comorbidities and extreme frailty, for whom a transition to palliative care might be prudent. Selecting nonagenarian patients with low comorbidity index and with no extreme frailty, adopting a minimalistic approach and paying attention to vascular access hemostasis may provide the elements that may lead to a successful, desirable and hopefully cost-effective outcome.
\end{abstract}

Keywords: Elderly; nonagenarians; aortic stenosis; transcatheter aortic valve implantation (TAVI); aortic valve replacement (AVR); frailty; comorbidities; quality of life (QoL); cost-efficacy

Submitted Jun 09, 2017. Accepted for publication Jun 27, 2017.

doi: $10.21037 /$ acs.2017.07.11

View this article at: http://dx.doi.org/10.21037/acs.2017.07.11

\section{Introduction}

As life expectancy increases, the number of nonagenarians grows. In the US, the 90 -and-older population nearly tripled over the past three decades, reaching 1.9 million in 2010, comprising $4.7 \%$ of the older population (age 65 and older) in 2011, as compared with only $2.8 \%$ in 1980 (1). Over the next three to four decades, this population is projected to more than quadruple, with its share likely to reach $10 \%$ by 2050 . The greatest proportion are nonagenarians; however, a small portion of this population comprises increasing numbers of centenarians, projected to reach almost 850,000 in the US by the middle of the century (2). The majority of people 90 and older report having one or more disabilities, are more likely to be women and to have higher widowhood, poverty and disability rates than younger people. It is in this patient population with an increased prevalence of aortic stenosis that cardiologists and cardiac surgeons will be more frequently confronted with the decision dilemma of conservative versus interventional treatment, trying to balance perils, cost, and benefits.

Although some investigators have proposed that transcatheter aortic valve implantation (TAVI) remains an option for the treatment of severe symptomatic aortic 
stenosis for the elderly regardless of their age (3), TAVI in nonagenarians may have a satisfactory outcome only when those with a healthier profile are selected for the procedure (4). Other investigators have proposed that in nonagenarians, both TAVI and surgical aortic valve replacement (AVR) can be performed with acceptable inhospital outcomes and that interventional management should not be precluded based on age alone (5).

According to the Society of Thoracic Surgeons (STS)/ American College of Cardiology (ACC) Transcatheter Valve Therapy Registry, in the U.S. clinical practice, approximately $16 \%$ of patients undergoing TAVI are nonagenarians, in whom the 30 -day $(8.8 \%$ vs. $5.9 \%)$ and 1 -year mortality rates $(24.8 \%$ vs. $22 \%)$ are statistically modestly higher compared with younger patients undergoing TAVI (6). Nevertheless, as of to date, the few reports of TAVI results in nonagenarians point out several issues in this high-risk population (4-22) (Table 1). TAVI in a select group of nonagenarians is feasible with satisfactory short- and medium-term outcomes. However, procedural complication rate is higher, as well as 30-day and 1-3-year mortality rates compared with younger patients; there is an apparent relation of less satisfactory outcomes with a higher comorbidity index. Unfortunately, there is a dearth of quality-of-life and cost-effectiveness data.

\section{Transfemoral versus other route}

According to a study reporting short- and mid-term outcomes of 531 nonagenarian patients undergoing transfemoral $(n=329)$ or transapical $(n=202)$ TAVI in the PARTNER-I trial (17), the post-procedure 30-day stroke risk was $3.6 \%$ vs. $2 \%$ for the two groups; major adverse events occurred in $35 \%$ vs. $32 \%$ of patients; 30 -day paravalvular leak was greater than moderate in $1.4 \%$ $v s$. $0.61 \%$; median post-procedure length of stay was $5 v$. 8 days. Thirty-day mortality was $4 \%$ vs. $12 \%$ and 3 -year mortality $48 \%$ vs. $54 \%$ ( $44 \%$ vs. $42 \%$ for the matched population). By 6 months, most quality of life (QoL) measures had stabilized at a better level than baseline, for both groups. The authors concluded that age alone should not preclude referral for TAVI, as TAVI can be performed in nonagenarians with acceptable short- and mid-term outcomes; transapical TAVI appears to carry a higher risk of early death without a difference in intermediateterm mortality. Nevertheless, the poorer outcome of the transapical approach has been confirmed by many other studies, as well $(15,17,21,22)$. Indeed, routes other that the transfemoral approach seem to be of much higher risk and probably futile (15).

\section{Frailty}

Frailty appears to improve the identification of high-risk patients beyond known risk scores and thus it has been included as a high-risk factor for aortic valve surgery, favoring the selection of a transcatheter approach (23). However, frailty has also been shown to confer strong predictive ability for both short- and long-term mortality after TAVI (24). Among 75 nonagenarians undergoing TAVI, frail patients had higher 30 -day (14\% vs. $2 \%$; $\mathrm{P}=0.059)$ and 2-year mortality ( $31 \%$ vs. 9\%; $\mathrm{P}=0.018)$ (25). Kaplan-Meier analysis estimated all-cause mortality to be significantly higher in the frail group (log-rank test; $\mathrm{P}=0.042)$. Frailty status was independently associated with increased mortality [hazard ratio (HR): $1.84 ; \mathrm{P}=0.028$ ] after TAVI. Other studies have also shown significant associations between frailty indices and 1-year all-cause mortality after TAVI $(26,27)$. An incremental rise in mortality and post-TAVI length of stay with increasing frailty has been reported with dismal prognosis in extremely frail patients (28). Thus, there appears to be a critical role of frailty in identifying patients unlikely to benefit from TAVI (29).

\section{Comorbidities}

Over $80 \%$ of nonagenarians who live in households or noninstitutional groups and virtually all (98\%) of those residing in institutional group quarters (e.g., nursing homes) have some type of disability (1). Similarly, the majority $(\sim 2 / 3)$ of nonagenarians with severe aortic stenosis have several comorbidities (30). Among 177 (117 females) nonagenarians with severe aortic stenosis (mean age 91.1 years), only 56 patients $(31.6 \%)$ had a low (<3) Charlson comorbidity index. A strong association between comorbidity and oneyear overall mortality was observed, with higher oneyear mortality in patients with Charlson index $\geq 3$ (66.4\% vs. $32.1 \%, \mathrm{P}<0.001)$. A total of 150 patients $(84.7 \%)$ were managed conservatively, and 27 (15.3\%) underwent TAVI. Predictors of a conservative management were treatment outside of TAVI centers, lower mean aortic gradient and better functional class.

One of the major comorbidities is chronic kidney disease (CKD). According to a recent multicenter observational study of 1904 patients undergoing TAVI, 421 patients were 


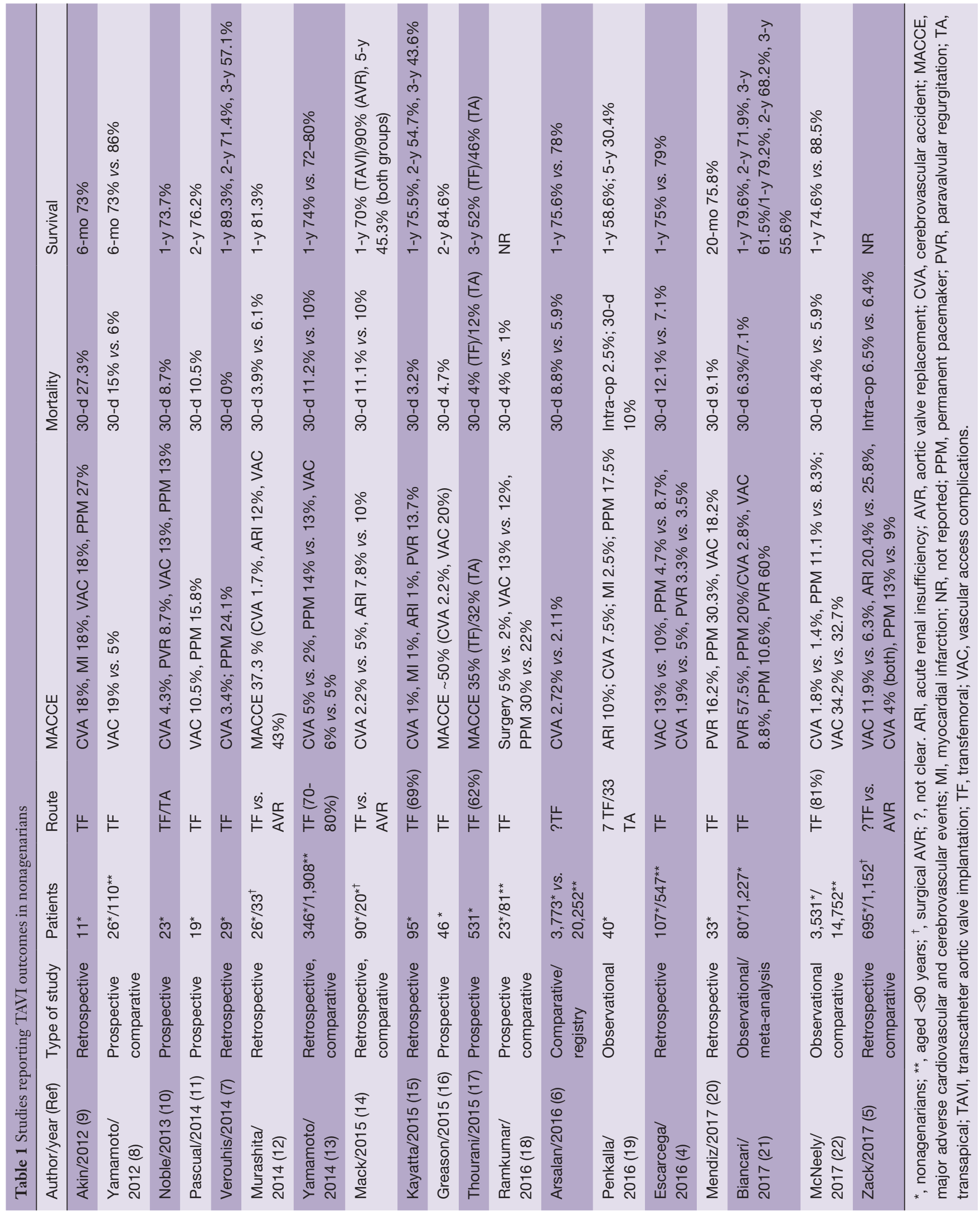


staged S5 $(\mathrm{n}=74)$ or S4 $(\mathrm{n}=347)$ with estimated glomerular filtration rate of $15-29 \mathrm{~mL} / \mathrm{min} / 1.73 \mathrm{~m}^{2}$ for stage 4 (S4), and $<15 \mathrm{~mL} / \mathrm{min} / 1.73 \mathrm{~m}^{2}$ for stage 5 (S5) CKD (31). Periprocedural and 30-day outcomes were similar in S5 and S4 patients. During a mean of 1.8 years, S5 patients had higher mortality rates $(69 \%$ vs. $39 \%, \mathrm{P}<0.01)$ and cardiac death (19\% vs. 9\%, $\mathrm{P}=0.02)$ compared with $\mathrm{S} 4$ patients. Male gender (HR 1.6), left ventricular ejection fraction $<30 \%$ (HR 2.3), atrial fibrillation (HR 1.4), and S5 CKD (HR 1.5) were independent predictors of death. Thus, in pre-dialytic or dialytic CKD patients (S5), TAVI appears futile with very poor outcomes even when performed not necessarily in nonagenarians, although in severe CKD (S4) patients, a more rigorous selection is required to avoid such outcomes. Indeed, other studies have confirmed that preprocedural severe CKD is a significant predictor for 1-year mortality in TAVI patients (32).

The accumulated evidence over the years shows that most cardiac surgical procedures can be performed in the elderly with very satisfactory outcomes. However, the risk in these patients for such procedures is only deemed acceptable in the absence of comorbidities (33). This holds true for TAVI procedures as well (34). Among comorbidities, cerebrovascular disease, CKD and frailty appear to confer a worse outcome in the very elderly (23,30-32). Careful patient selection, a lege artis procedure, a minimalistic approach and vascular hemostasis are of utmost importance for a successful TAVI procedure $(35,36)$.

\section{Complications}

Although procedural complications are encountered in all TAVI procedures, the very elderly appear to be more prone to complications. Sudden development of hypotension is the most worrisome of all. Such a development should prompt exclusion of five major pathologies (perilous pentad) (37); retroperitoneal bleeding from access site rupture that may develop at different times during the procedure; aortic dissection or rupture, pericardial tamponade, coronary ostial obstruction, or acute severe aortic regurgitation that may develop with manipulations during valve crossing, balloon dilatation, and/or prosthetic valve deployment.

With regards to post-procedural complications, according to a systematic review and meta-analysis, in general, multiorgan failure, heart failure, and vascular complications appear to be leading causes of death for TAVI within 30 days (38). Beyond 30 days, infection/sepsis, heart failure, and sudden death appear to predominate.
TAVI in high-risk patients (mean STS score $>8$ ), including nonagenarians, incurs higher rate of cardiovascular deaths after 30 days compared to moderate risk (STS score 4-8) patients (56\% vs. $33.5 \%, \mathrm{P}=0.005)$.

\section{Paravalvular regurgitation}

An important and serious procedural problem in this group of patients is the rate of moderate/severe paravalvular regurgitation which is exceedingly high, reaching levels of $\sim 17-60 \%(20,21)$. As expected in these very elderly patients, the almost ubiquitous presence of severe aortic valve calcification predicts the development of this complication. Unfortunately, this has been shown to be associated with poor TAVI outcome and increased mortality (39).

\section{Clinical outcomes}

Data from the STS/ACC Transcatheter Valve Therapies Registry on 12,182 patients (mean age 84 years), who underwent TAVI [2011-2013], indicated that only $60 \%$ were discharged to home (40). The 30-day mortality was $7 \%$, the stroke rate $\sim 4 \%$ and 1 year survival was $\sim 76 \%$. Factors associated with 1-year mortality included advanced age (HR for $\geq 95$ vs. $<75$ years, 1.61; HR for $85-$ $94 v s .<75$ years, 1.35; and HR for 75-84 vs. $<75$ years, 1.23), male gender (HR, 1.21), end-stage renal disease (HR, 1.66), severe chronic obstructive pulmonary disease (HR, 1.39), non-transfemoral access (HR, 1.37), STS score $>15 \%$ s. $<8 \%$ (HR, 1.82), and preoperative atrial fibrillation/flutter (HR, 1.37). These findings should be helpful in comparing TAVI outcomes in nonagenarians with younger patients.

As deduced from studies reporting results of TAVI in nonagenarians summarized in Table 1, TAVI, as long as it is performed via the transfemoral route, is feasible and relatively safe and effective in this patient subpopulation, albeit at higher risk compared to younger patients, with satisfactory short- and mid-term outcomes, however, long-term data are meagre (4-22). Procedural and 30day mortality ranges from $0 \%$ to $27 \%$, hovering around $10 \%$, stroke risk ranges from $2 \%$ to $18 \%$ (average $3-4 \%$ ), bleeding and vascular complications range from $9 \%$ to $34 \%$ (average $16 \%$ ), renal insufficiency ranges from $1 \%$ to $10 \%$, while the emerging need for a pacemaker has a wide range depending on the type of valve employed (5-30\%) (41). Unfortunately, paravalvular regurgitation is inordinately high in this group (7-60\%).

As long as the nonagenarians who are undergoing TAVI 


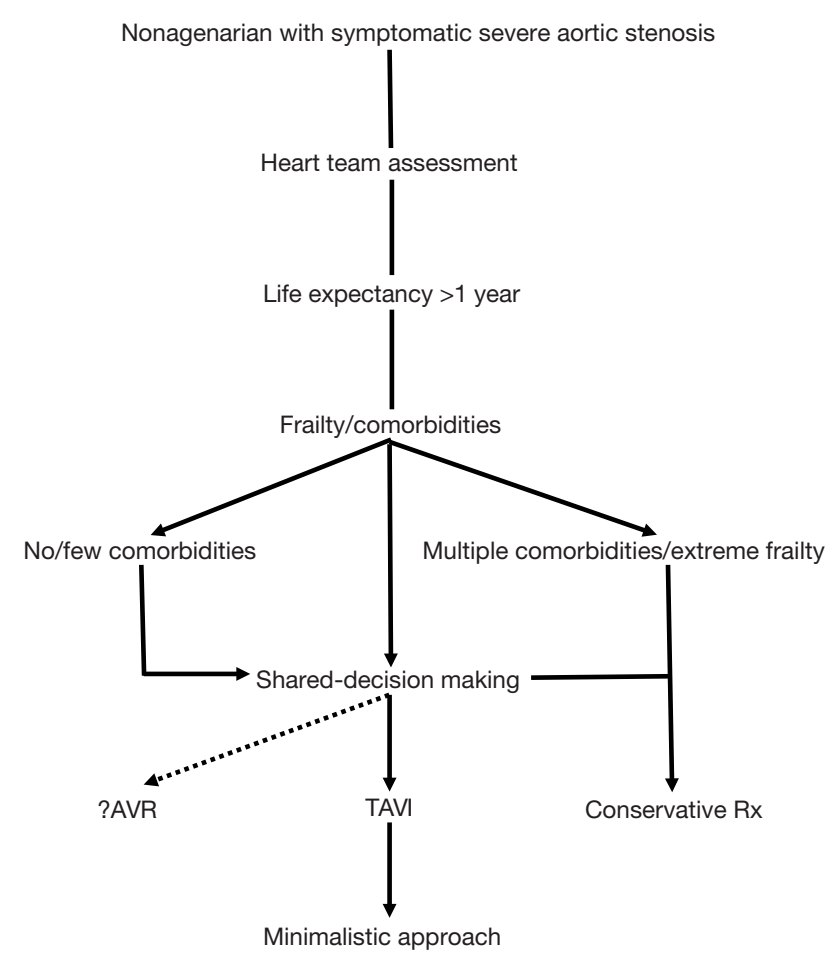

(TF/local anesthesia \pm conscious sedation/vessel pre-closure/no TEE) No balloon pre-dilation (if possible)/minimize PVR Preparedness to manage complications

Figure 1 The proposed algorithm for TAVI selection in a nonagenarian patient starts with the Heart Team assessment individualized for each patient by involving patient and family in a realistic approach aiming at minimizing procedural risk and enhancing patient benefit and welfare (see text for discussion). AVR, (surgical) aortic valve replacement; PVR, paravalvular regurgitation; Rx, treatment; TAVI, transcatheter aortic valve implantation; TEE, transesophageal echocardiography; TF, transfemoral.

belong to the inoperable or very high-risk group for surgery with life expectancy of at least 1-year, a mean 74\% 1-year survival of this group (Table 1) compares well to the $76 \%$ 1-year survival rate of the whole TAVI population (40) and lends support for this procedure in this cohort, given the prognosis of untreated severe aortic stenosis. Approximately one-half of the patients in the PARTNER B cohort that were randomly assigned to the medical therapy arm had expired by 1 year (42). However, there are other issues which are equally important when considering such an intervention in this extreme-aged population. Among other problems, the high intraprocedural risk comprising higher rates of mortality and other major adverse cardiovascular and cerebrovascular events (stroke, vascular access complications, paravalvular regurgitation, heart failure, renal insufficiency, infection/sepsis and need for permanent pacemaker implantation) may significantly detract from the value of this intervention.

Finally, appropriate patient selection apparently plays a crucial role by determining the associated comorbidities and assisting both the physicians and the patient/family in the shared-decision making process (43) (Figure 1). Importantly, issues such as QoL and cost, which have not been adequately examined in the studies, need also be addressed in this process. As analyses of large cohort studies indicate that about one third of patients experience a poor outcome at 6 months following TAVI, models have been developed and proposed to identify patients at high risk for such poor outcomes after TAVI to help decision making by guiding therapeutic options and offering patients realistic expectations of long-term outcomes $(29,44)$.

\section{Cost}

As the cost of TAVI is currently very high and much higher than surgical AVR (45), one may argue that based on the equivalent results presented by some investigators for TAVI and surgical AVR in nonagenarians, referral for AVR in these patients should not be precluded based on age alone (5).

A minimalist approach, with use of local anesthesia and without intra-procedural transesophageal echo guidance, may compensate for and mitigate some of the TAVI cost without apparently compromising safety and efficacy (46).

However, cost-effectiveness studies are lacking in this subpopulation. Nevertheless, based on such studies in the general TAVI population, whereby this procedure is costeffective with an acceptable incremental cost-effectiveness ratio (ICER) $(\leq \$ 50,000$ for the US standards) only for the inoperable and borderline for the high-risk patients (45), some suggestions are emerging that the ICER for the nonagenarians may be high above the society's willing-topay threshold (47).

\section{QoL}

Improvement in QoL is an important measure of procedural outcomes. However, there is a dearth of QoL data in the TAVI population and particularly in the nonagenarian subpopulation. According with a world-wide survey of current practices in TAVI, frailty (44\%) and QoL 
(28\%) assessments were less frequently performed (48). It has also been shown that frailty is independently associated with discharge to a rehabilitation facility (49), a factor that apparently impairs QoL.

According to QoL data from the German TAVI registry (50), among 2,288 patients undergoing TAVI, only $20 \%$ of the surviving patients derived QoL benefits at one year after TAVI, with $40-60 \%$ presenting no change in QoL status, while a worrisome $20 \%$ rate of worsening was recorded. In another QoL study comprising 7,014 surviving TAVI patients, two thirds had a QoL benefit at 1 year, however still 1 in 3 patients had a poor QoL outcome at 1 year (51).

Regarding the nonagenarian group, data from the STS/ACC registry in 3,773 patients indicate a significant increase in QoL scores by 30 days, which were though significantly lower in nonagenarians compared with younger patients (6). Nevertheless, by 1 year, there were no differences in QOL between age groups. The authors propose that these findings suggest that nonagenarians may recover more slowly after TAVI and thus need more time until the beneficial effect of the procedure is appreciable. In another QoL data analysis in 531 nonagenarians from the PARTNER I trial, the authors reported that by 6 months, most QoL measures had stabilized at a level considerably better than baseline (17).

\section{Conclusions}

Although chronologic age alone should not deprive patients from TAVI, it is the physiologic age that should be factored in a shared-decision making approach (participatory medicine) to determine a patient's candidacy for this nonsurgical therapeutic modality. Thus, it practically comes down to the patient selection process by having the heart team review each case individually and getting the patient and the family involved (43) (Figure 1). The aim is to make the patient feel better and live longer. Specifically, with regards to the nonagenarian group, patient preferences and values should be taken into account after having apprised the patient and the family with all the procedure specifics, sharing and respecting goals, expectations and end-of-life views and ideas. All parties involved should be cognizant of the fact that this extreme-aged group has already surpassed the limits of life expectancy and decisions about the procedure come at a cost which may not always and necessarily be viewed upon favorably depending on the willingness-to-pay threshold of the society. There is always the realistic possibility that TAVI may be clinically futile for patients who have a multitude of comorbidities and extreme frailty, for whom a transition to palliative care might be prudent (52). Selecting nonagenarian patients with low comorbidity index and with no extreme frailty, adopting a minimalistic approach and paying attention to vascular access hemostasis may provide the elements that lead to a successful, desirable and hopefully cost-effective outcome (Figure 1). Finally, quality-of-life studies are direly needed and eagerly awaited in this patient subpopulation in order to provide guidance in the patient selection process.

\section{Acknowledgements}

None.

\section{Footnote}

Conflicts of Interest: The authors have no conflicts of interest to declare.

\section{References}

1. He W, Muenchrath MN. U.S. Census Bureau, American Community Survey Reports, ACS-17, 90+ in the United States: 2006-2008, U.S. Government Printing Office, Washington, DC, 2011.

2. Krach CA, Velkoff VA. U.S. Bureau of the Census, Current Population Reports, Series P23-199RV Centenarians in the United States. U.S. Government Printing Office, Washington, 1999.

3. Attizzani GF, Ohno Y, Latib A, et al. Age-Related Differences in 1- and 12-Month Outcomes in Patients Undergoing Transcatheter Aortic Valve Implantation (from a Large Multicenter Data Repository). Am J Cardiol 2016;118:1024-30.

4. Escarcega RO, Baker NC, Lipinski MJ, et al. Clinical profiles and correlates of mortality in nonagenarians with severe aortic stenosis undergoing transcatheter aortic valve replacement. Am Heart J 2016;173:118-25.

5. Zack CJ, Al-Qahtani F, Kawsara A, et al. Comparative Outcomes of Surgical and Transcatheter Aortic Valve Replacement for Aortic Stenosis in Nonagenarians. Am J Cardiol 2017;119:893-9.

6. Arsalan M, Szerlip M, Vemulapalli S, et al. Should Transcatheter Aortic Valve Replacement Be Performed in Nonagenarians?: Insights From the STS/ACC TVT Registry. J Am Coll Cardiol 2016;67:1387-95. 
7. Verouhis D, Yamasaki K, Ivert T, et al. Transcatheter aortic valve implantation is feasible and safe in nonagenarians. J Am Geriatr Soc 2014;62:189-90.

8. Yamamoto M, Meguro K, Mouillet G, et al. Comparison of effectiveness and safety of transcatheter aortic valve implantation in patients aged $>/=90$ years versus $<90$ years. Am J Cardiol 2012;110:1156-63.

9. Akin I, Kische S, Paranskaya L, et al. Morbidity and mortality of nonagenarians undergoing CoreValve implantation. BMC Cardiovasc Disord 2012;12:80.

10. Noble S, Frangos E, Samaras N, et al. Transcatheter aortic valve implantation in nonagenarians: effective and safe. Eur J Intern Med 2013;24:750-5.

11. Pascual I, Lopez-Otero D, Munoz-Garcia AJ, et al. Safety and efficacy of transcatheter aortic valve implantation in nonagenarian patients. Rev Esp Cardiol (Engl Ed) 2014;67:583-4.

12. Murashita T, Greason KL, Suri RM, et al. Aortic valve replacement for severe aortic valve stenosis in the nonagenarian patient. Ann Thorac Surg 2014;98:1593-7.

13. Yamamoto M, Mouillet G, Meguro K, et al. Clinical results of transcatheter aortic valve implantation in octogenarians and nonagenarians: insights from the FRANCE-2 registry. Ann Thorac Surg 2014;97:29-36.

14. Mack MC, Szerlip M, Herbert MA, et al. Outcomes of Treatment of Nonagenarians With Severe Aortic Stenosis. Ann Thorac Surg 2015;100:74-80.

15. Kayatta MO, Thourani VH, Jensen HA, et al. Outcomes for Transcatheter Aortic Valve Replacement in Nonagenarians. Ann Thorac Surg 2015;100:1261-7; discussion 1267.

16. Greason KL, Pochettino A, Sandhu GS, et al. Transcatheter aortic valve insertion in the nonagenarian patient. J Thorac Cardiovasc Surg 2015;150:720-1.

17. Thourani VH, Jensen HA, Babaliaros V, et al. Outcomes in Nonagenarians Undergoing Transcatheter Aortic Valve Replacement in the PARTNER-I Trial. Ann Thorac Surg 2015;100:785-92; discussion 793.

18. Ramkumar S, Rashid HN, Zaman S, et al. Feasibility and clinical outcomes in nonagenarians undergoing transcatheter aortic valve replacement with the LOTUS valve. J Geriatr Cardiol 2016;13:636-8.

19. Penkalla A, Kempfert J, Unbehaun A, et al. Transcatheter Aortic Valve Implantation in Nonagenarians. Innovations (Phila) 2016;11:390-5.

20. Mendiz O, Fava C, Cura F, et al. Clinical outcome in nonagenarians undergoing transcatheter valve replacement. Indian Heart J 2017. [Epub ahead of print].
21. Biancari F, D'Errigo P, Rosato S, et al. Transcatheter aortic valve replacement in nonagenarians: early and intermediate outcome from the OBSERVANT study and meta-analysis of the literature. Heart Vessels 2017;32:157-65.

22. McNeely C, Zajarias A, Robbs R, et al. Transcatheter Aortic Valve Replacement Outcomes in Nonagenarians Stratified by Transfemoral and Transapical Approach. Ann Thorac Surg 2017;103:1808-14.

23. Graham A, Brown CH. Frailty, Aging, and Cardiovascular Surgery. Anesth Analg 2017;124:1053-60.

24. Thongprayoon C, Cheungpasitporn $\mathrm{W}$, Thamcharoen $\mathrm{N}$, et al. Association of frailty status with acute kidney injury and mortality after transcatheter aortic valve replacement: A systematic review and meta-analysis. PLoS One 2017;12:e0177157.

25. Okoh AK, Chauhan D, Kang N, et al. The impact of frailty status on clinical and functional outcomes after transcatheter aortic valve replacement in nonagenarians with severe aortic stenosis. Catheter Cardiovasc Interv 2017. [Epub ahead of print].

26. Kleczynski P, Dziewierz A, Bagienski M, et al. Impact of frailty on mortality after transcatheter aortic valve implantation. Am Heart J 2017;185:52-8.

27. Green P, Arnold SV, Cohen DJ, et al. Relation of frailty to outcomes after transcatheter aortic valve replacement (from the PARTNER trial). Am J Cardiol 2015;116:264-9.

28. Chauhan D, Haik N, Merlo A, et al. Quantitative increase in frailty is associated with diminished survival after transcatheter aortic valve replacement. Am Heart J 2016;182:146-54.

29. Puri R, Iung B, Cohen DJ, et al. TAVI or No TAVI: identifying patients unlikely to benefit from transcatheter aortic valve implantation. Eur Heart J 2016;37:2217-25.

30. Bernal E, Ariza-Sole A, Bayes-Genis A, et al. Management of Nonagenarian Patients With Severe Aortic Stenosis: The Role of Comorbidity. Heart Lung Circ 2017. [Epub ahead of print].

31. Conrotto F, Salizzoni S, Andreis A, et al. Transcatheter Aortic Valve Implantation in Patients With Advanced Chronic Kidney Disease. Am J Cardiol 2017;119:1438-42.

32. Thourani VH, Forcillo J, Beohar N, et al. Impact of Preoperative Chronic Kidney Disease in 2,531 High-Risk and Inoperable Patients Undergoing Transcatheter Aortic Valve Replacement in the PARTNER Trial. Ann Thorac Surg 2016;102:1172-80.

33. Nicolini F, Agostinelli A, Vezzani A, et al. The evolution of cardiovascular surgery in elderly patient: a review 
of current options and outcomes. Biomed Res Int 2014;2014:736298.

34. Vahl TP, Kodali SK, Leon MB. Transcatheter Aortic Valve Replacement 2016: A Modern-Day "Through the Looking-Glass" Adventure. J Am Coll Cardiol 2016;67:1472-87.

35. Babaliaros V, Devireddy C, Lerakis S, et al. Comparison of transfemoral transcatheter aortic valve replacement performed in the catheterization laboratory (minimalist approach) versus hybrid operating room (standard approach): outcomes and cost analysis. JACC Cardiovasc Interv 2014;7:898-904.

36. Manolis AS, Polydorou A, Rouska E, et al. Transcatheter aortic valve iplantation in severe left ventricular dysfunction: A viable option in a patient with low-flow, low-gradient critical aortic stenosis. Hosp Chronicles 2013;8:97-9.

37. El-Gamel A. Cardiovascular Collapse During Transcatheter Aortic Valve Replacement: Diagnosis and Treatment of the "Perilous Pentad". Aorta (Stamford) 2013;1:276-82.

38. Xiong TY, Liao YB, Zhao ZG, et al. Causes of Death Following Transcatheter Aortic Valve Replacement: A Systematic Review and Meta-Analysis. J Am Heart Assoc 2015;4:e002096.

39. Dahou A, Ribeiro HB, Rodes-Cabau J, et al. Impact and Management of Paravalvular Regurgitation After Transcatheter Aortic Valve Replacement. Interv Cardiol Clin 2015;4:67-82.

40. Holmes DR, Jr., Brennan JM, Rumsfeld JS, et al. Clinical outcomes at 1 year following transcatheter aortic valve replacement. JAMA 2015;313:1019-28.

41. Manolis AS. Need for a permanent pacemaker after transcatheter aortic valve implantation (TAVI). Rhythmos 2016;11:87-9.

42. Leon MB, Smith CR, Mack M, et al. Transcatheter aortic-valve implantation for aortic stenosis in patients who cannot undergo surgery. N Engl J Med

Cite this article as: Manolis AS, Manolis AA. Transcatheter aortic valve implantation in nonagenarians: selectively feasible or extravagantly futile? Ann Cardiothorac Surg 2017;6(5):524531. doi: 10.21037/acs.2017.07.11
2010;363:1597-607.

43. Otto CM, Kumbhani DJ, Alexander KP, et al. 2017 ACC Expert Consensus Decision Pathway for Transcatheter Aortic Valve Replacement in the Management of Adults With Aortic Stenosis: A Report of the American College of Cardiology Task Force on Clinical Expert Consensus Documents. J Am Coll Cardiol 2017;69:1313-46.

44. Arnold SV, Afilalo J, Spertus JA, et al. Prediction of Poor Outcome After Transcatheter Aortic Valve Replacement. J Am Coll Cardiol 2016;68:1868-77.

45. Manolis AS. Transcatheter aortic valve implantation economics: a grisly reality. Ann Cardiothorac Surg 2017. [Epub ahead of print].

46. Jabbar A, Khurana A, Mohammed A, et al. Local Versus General Anesthesia in Transcatheter Aortic Valve Replacement. Am J Cardiol 2016;118:1712-6.

47. Weintraub WS. TAVR in Nonagenarians: Pushing the Boundaries. J Am Coll Cardiol 2016;67:1396-8.

48. Cerrato E, Nombela-Franco L, Nazif TM, et al. Evaluation of current practices in transcatheter aortic valve implantation: The WRITTEN (WoRldwIde TAVI ExperieNce) survey. Int J Cardiol 2017;228:640-7.

49. Huded CP, Huded JM, Friedman JL, et al. Frailty Status and Outcomes After Transcatheter Aortic Valve Implantation. Am J Cardiol 2016;117:1966-71.

50. Lange R, Beckmann A, Neumann T, et al. Quality of Life After Transcatheter Aortic Valve Replacement: Prospective Data From GARY (German Aortic Valve Registry). JACC Cardiovasc Interv 2016;9:2541-54.

51. Arnold SV, Spertus JA, Vemulapalli S, et al. Qualityof-Life Outcomes After Transcatheter Aortic Valve Replacement in an Unselected Population: A Report From the STS/ACC Transcatheter Valve Therapy Registry. JAMA Cardiol 2017;2:409-16.

52. Lauck SB, Gibson JA, Baumbusch J, et al. Transition to palliative care when transcatheter aortic valve implantation is not an option: opportunities and recommendations. Curr Opin Support Palliat Care 2016;10:18-23. 